\title{
Desempenho do e-reader lev: uma análise utilizando a ferramenta eye tracking
}

Performance of the lev e-reader: an analysis using the eye tracking tool

Márcia Cattoi Schmidt ${ }^{1}$

Diogo Gonçalves Martins ${ }^{2}$

Ricardo Schwinn Rodrigues ${ }^{3}$

Aline Girardi Gobbi ${ }^{4}$

Giselle Schmidt Alves Diaz Merino ${ }^{5}$

Marcelo Gitirana Gomes Ferreira ${ }^{6}$ 


\section{Resumo}

Acompanhando a evolução dos leitores eletrônicos, sua tecnologia e problemas decorrentes, o presente estudo buscou levantar dados a respeito da usabilidade do produto LEV, e-reader da empresa Saraiva. Dotado de uma aplicabilidade restrita apenas à leitura de livros digitais, o estudo da eficácia do dispositivo detectou uma não correspondência de expectativas quanto à interface em alguns participantes. Utilizouse a ferramenta Eye Tracking para medições mais completas no experimento, onde onze participantes realizaram seis tarefas determinadas. Os resultados apontam que os usuários têm uma grande expectativa de que o sistema siga a lógica e tenha o mesmo tempo de resposta de outros produtos de leitura similares.

Palavras-chave: interface, usabilidade, eye-tracking, e-reader, design.

\section{Abstract}

Following the evolution of electronic readers devices, its technology and problems arise, this study aimed to collect data about the usability of LEV device, an e-reader by Saraiva. Endowed with a limited applicability for digital books reading only, the device's efficacy study detected a mismatch of expectations regarding the interface in some participants. We used the Eye Tracking tool for more comprehensive measurements in the experiment, in which eleven participants performed six specific tasks. The results show that users have a great expectation that the system's logic will follow, and have the same response time, of other similar reading devices.

Key-words: interface, usability, eye-tracking, e-reader, design.

ISSN: 2316.7963
4 Doutoranda, Universidade Federal de Santa Catarina UFSC, Florianópolis, SC, Brasil. aline.gobbi@gmail.com

5 Prof. Dra, Universidade Federal de Santa Catarina UFSC, Florianópolis, SC, Brasil. gisellemerino@gmail.com

6 Prof. Dr. Universidade do Estado de Santa Catarina UDESC, Florianópolis, SC, Brasil. marcelo.gitirana@gmail.com

Márcia Cattoi Schmidt, Diogo Gonçalves Martins, Ricardo Schwinn Rodrigues, Aline Girardi Gobbi, Giselle Schmidt Alves Diaz Merino e Marcelo Gitirana Gomes Ferreira 


\section{Introdução}

Os estudos a respeito do mercado editorial brasileiro indicam queda no interesse dos brasileiros pela leitura, como o do Instituto Pró-Livro, que realiza inquirição periódica com uma amostra representativa de todos os estados brasileiros. A edição de 2016 contou com a participação de 5.012 indivíduos. A pesquisa identifica a penetrabilidade da leitura de livros no Brasil e o acesso a livros, além de preferências, perfis dos leitores e não leitores, e as barreiras para o crescimento da leitura no Brasil.

Muitos editores viram nos livros digitais uma nova opção para o problema da inércia ou do decrescimento na leitura. Pesquisas comerciais mostram que não só o novo formato editorial nunca alcançou o sucesso no Brasil, como também estagnou nos Estados Unidos, mercado onde a venda de livros digitais vinha aumentando nos últimos anos (CUNHA, 2016). Com o advento dos e-readers e o incremento das mídias digitais no final dos anos 1990, e dos tablets no início dos anos 2000, muito se falou na possível mudança positiva dos hábitos de leitura, fato que nunca se confirmou (O GLOBO, 2015). A pesquisa baseia-se na hipótese de que a queda do mercado pode estar vinculada à insatisfação do usuário quanto à usabilidade dos e-readers disponíveis, e isto pode ocorrer quando a evolução da tecnologia está centrada na máquina e não no usuário.

\section{Objetivos}

O experimento foi realizado com o propósito de levantar dados sobre: o uso intuitivo do produto e-reader LEV (Saraiva), o nível de satisfação do usuário durante o uso e a identificação de problemas de interface no produto.

\section{Sobre dispositivos e-reader}

Hoje, quando se fala em livros digitais, é possível citar duas categorias de aparelhos que possibilitam a sua leitura, os dedicados e os não dedicados. $O$ dedicado refere-se ao aparelho que é específico para este fim, como é o caso do e-reader. Os não dedicados são os desktops, os tablets e os smartphones, que, embora permitam a leitura, não foram concebidos com apenas esta finalidade (ZAMBARBIERI e CARNIGLIA, 2012).

Com o objetivo de tentar explicar a expectativa frustrada da realidade dos livros digitais, muitos autores pesquisaram sobre as diferenças da leitura nos livros impressos, nos aparelhos dedicados e não dedicados, procurando algum indício da não aceitação dos usuários para esta nova tecnologia.

Siegenthaler, juntamente com outros pesquisadores, realizaram estudos sobre o assunto: Siegenthaler et al (2010) compararam a leitura realizada com e-reader (que utiliza tecnologia de display e-ink) e tablet (que utiliza a tecnologia de LCD para display) em longos períodos de leitura. As telas e-ink são formadas por duas camadas transparentes com microesferas brancas e pretas entre elas, responsáveis pela produção da imagem. Os autores concluíram que, utilizando a fadiga visual como 
parâmetro, os dois aparelhos se mostraram bons para longos períodos de leitura, contrapondo-se a uma hipótese existente sobre o desconforto na leitura em mídias digitais. Em outro estudo, Siegenthaler et al (2011) registraram que o comportamento de leitura nos e-readers e nos livros impressos são muito parecidos. Nielsen (2010), em seu estudo comparativo entre dispositivos digitais e livros impressos, definiu que o tempo de leitura é menor $10,7 \%$ em e-readers e nos tablets é 6,2\% menor em comparação com a leitura dos livros impressos. Da mesma forma, autores como Gatsou et al (2015) exploraram a tecnologia emergente para um estudo comparativo entre dois dispositivos eletrônicos de leitura, um leitor dedicado e um não dedicado. Neste estudo em particular, os resultados mostram que os usuários realizaram as tarefas mais eficientemente no dispositivo não dedicado (multifuncional) em termos de medidas de desempenho, tais como: navegação, dificuldade da tarefa e satisfação.

Também há estudos como os de Larson (2008), que investigaram o uso de tecnologias digitais para ambientes de ensino, revelando por meio de um experimento com futuros professores, que a expectativa dos participantes passou de desfavorável para favorável, embora ainda preferissem o método impresso de leitura. A autora considera que a experiência com os futuros professores possibilitaria novas oportunidades de interação dos estudantes com a tecnologia, sendo para ela imperativa a nova alfabetização orquestrada pelos professores.

A escolha por este aparelho especificamente foi motivada por sua marca ser brasileira (Saraiva), e consequentemente apresentar mais títulos disponíveis em português até o momento. É neste cenário que foi realizado o presente trabalho, pela proposição de estudar a usabilidade do e-reader Lev, com o intuito de agregar mais uma importante informação rumo ao entendimento da realidade que emerge no mercado editorial.

\section{Materiais e métodos}

\subsection{Materiais}

O aparelho LEV utilizado é da empresa Saraiva, e tem as seguintes especificações técnicas: bateria de $1.800 \mathrm{MAH}$; conectividade Wi-Fi $802.11 \mathrm{~b} / \mathrm{g} / \mathrm{n}$ e Micro USB; dimensões: 166 x 120 × 9,4 mm; tela Touch HD de 758 x 1024px (213 dpi); leitura de formatos: ePUB, PDF, FB2, imagens (JPEG, GIF, PNG, BMP, ICO, TIFF e PSD) e texto (TXT e HTML); memória interna de 4 GB (2,8 GB disponíveis) ou 1.200 .000 páginas; memória externa com entrada para cartão microSD de até 32 GB MicroSDTM; peso de 190g; processador de $1 \mathrm{GHz}$; tela e-ink de 6 polegadas com resolução de $1024 \times 758$ pixels e iluminação integrada. Além das vantagens de mobilidade e armazenamento, também é possível comprar e receber e-books em minutos, fazer anotações e acessar notas de outros leitores, alterar tamanho de fonte, busca de palavras, dicionários, entre outras funções.

O equipamento utilizado para coleta de dados foi o modelo SMI Eye Tracking Glasses 2w (Senso Motoric Instruments) acompanhado do Software BeeGaze. Esta ferramenta capta os movimentos oculares por meio de óculos dotados de um conjunto de câmeras, revelando o mapeamento do olhar do usuário, entre outros dados 
quantitativos. O infravermelho cria reflexos na superfície do olho do participante (reflexo corneal), enquanto o sistema fornece análises para comparar a localização da reflexão na pupila do participante e áreas de maior interesse conforme o movimento dos olhos (ZAMBARBIERI; CARNIGLIA, 2012).

\subsection{Métodos}

O ambiente do teste foi o Núcleo de Gestão de Design e Laboratório de Design e Usabilidade (NGD/LDU) da Universidade Federal de Santa Catarina/UFSC. Foram selecionados onze universitários da UFSC, sem deficiência visual ou cognitiva, inexperientes no uso deste produto, sendo quatro do sexo masculino e sete do feminino. Faixas etárias: seis indivíduos de 18 a 28 anos completos, quatro indivíduos de 29 a 39 anos completos e um indivíduo de 40 anos completos ou mais. A coleta de dados ocorreu conforme a sequência:

1. O participante completou o questionário introdutório, e assinou o termo de consentimento.

2. Mostrou-se uma imagem ao participante contendo as principais funcionalidades do aparelho (Anexo 1).

3. O participante colocou o equipamento Eye Tracking e este foi calibrado.

4. O moderador leu ao participante cada tarefa a ser executada em voz alta, para que a realizasse seguindo o protocolo "Pensando em Voz Alta". A descrição das tarefas encontra-se no Quadro - Tarefas abaixo:

\begin{tabular}{|c|l|}
\hline Tarefa 1 & \multicolumn{1}{|c|}{ Encontrar o livro "Star Wars Aftermath" na memória do dispositivo. } \\
\hline Tarefa 2 & Aumentar e diminuir o zoom da página de leitura. \\
\hline Tarefa 3 & $\begin{array}{l}\text { Considerando que o manual do usuário é um livro, abrir e ir à página } \\
\text { 11, ler como procurar uma palavra no dicionário contido no aparelho. }\end{array}$ \\
\hline Tarefa 4 & $\begin{array}{l}\text { Na mesma página do manual, procurar o significado de uma palavra } \\
\text { qualquer no dicionário. }\end{array}$ \\
\hline Tarefa 5 & $\begin{array}{l}\text { Marcar um parágrafo do livro e fazer uma nota escrevendo a frase: } \\
\text { "teste de usabilidade". }\end{array}$ \\
\hline Tarefa 6 & $\begin{array}{l}\text { Abrir a loja de livros e colocar no "carrinho de compras" o livro "Harry } \\
\text { Potter e a Pedra Filosofal". }\end{array}$ \\
\hline
\end{tabular}

Quadro - Tarefas. Fonte - elaborado pelos autores.

5. Quando o participante considerar que realizou a tarefa, ela dirá em voz alta que terminou e seguirá à tarefa seguinte.

6. A tarefa será interrompida quando: 1) O participante declarar que não consegue realizar a tarefa, ou 2) O participante demorar mais do que 2 minutos tentando realizar a tarefa. Neste caso o moderador interromperá e solicitará que passe à tarefa seguinte.

7. Ao terminar todas as tarefas, encerra-se a gravação e solicita-se que o participante preencha o formulário SUS previamente preparado, além de questionário pós-teste. 


\section{Resultados e discussão}

A usabilidade de um produto é medida pela eficácia, eficiência e satisfação do usuário, de acordo com a norma da International Organization for Standardization (ISO) 9241-11. Para contemplar estes fatores, foram selecionadas seis tarefas com diferentes níveis de complexidade. A análise deu-se por: erro na tarefa (a partir de uma forma idealizada); tempo de execução; questionário pré e pós-teste; e escala SUS.

\subsection{Questão erro na tarefa}

Objetivando a análise das tarefas, foram definidas maneiras ideais de realizar cada uma delas, considerando o menor número possível de acionamentos até a conclusão da mesma. Esta forma "ideal" foi considerada como tarefa realizada com sucesso (S). Se o participante conseguiu realizar a tarefa, mas através de outro caminho, realizando mais acionamentos, a tarefa foi considerada realizada, mas com sucesso parcial $(P)$. Em caso de desistência do participante ou caso tenha sido necessária a ajuda do moderador para realizar a tarefa, esta foi considerada como tarefa falha (F). Os dados estão dispostos graficamente na Figura 1.

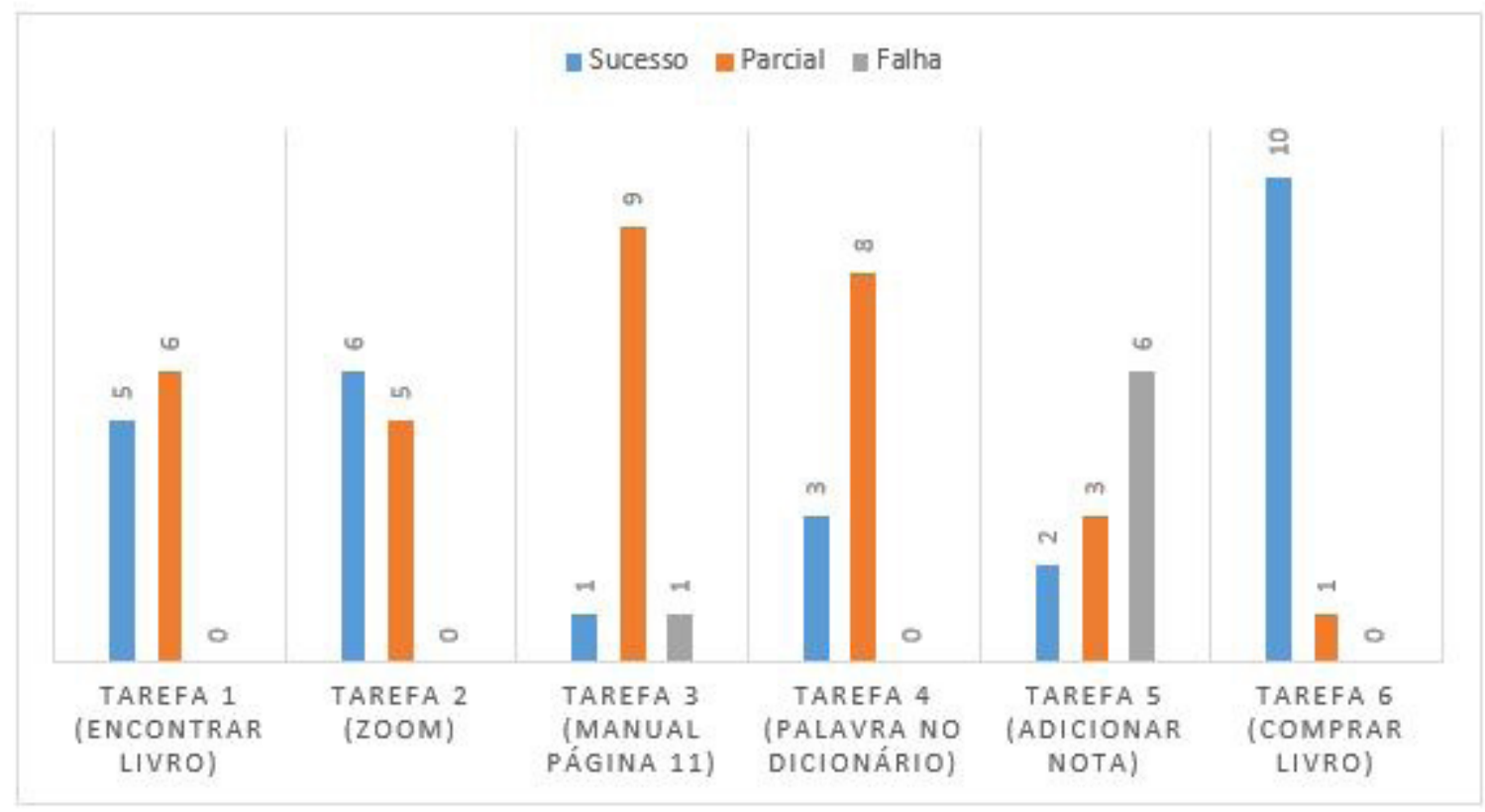

Figura 1 - Frequência dos acertos em relação às tarefas, por participante. Fonte - Elaborado pelos autores, conforme dados coletados.

\subsubsection{Tarefa 1 - Encontrar o livro}

Alguns dos participantes procuravam pelo livro específico direto na página inicial, em um espaço onde eram mostrados apenas alguns dos livros contidos na memória do aparelho. Este preview trata-se de uma pequena seleção aleatória sem qualquer critério aparente, com aleatoriedade comprovada em pós-teste pelos pesquisadores. Quando o livro solicitado estava neste espaço, os participantes chegaram até ele rapidamente. Quando não estava, o usuário poderia achar que o livro não está 
na memória do aparelho, realizando a tarefa em maior tempo. Considerou-se esta forma parcialmente correta. A maneira correta de realizar esta tarefa seria entrar em "Meus livros" no menu inferior, conforme Figura 2 e 3.

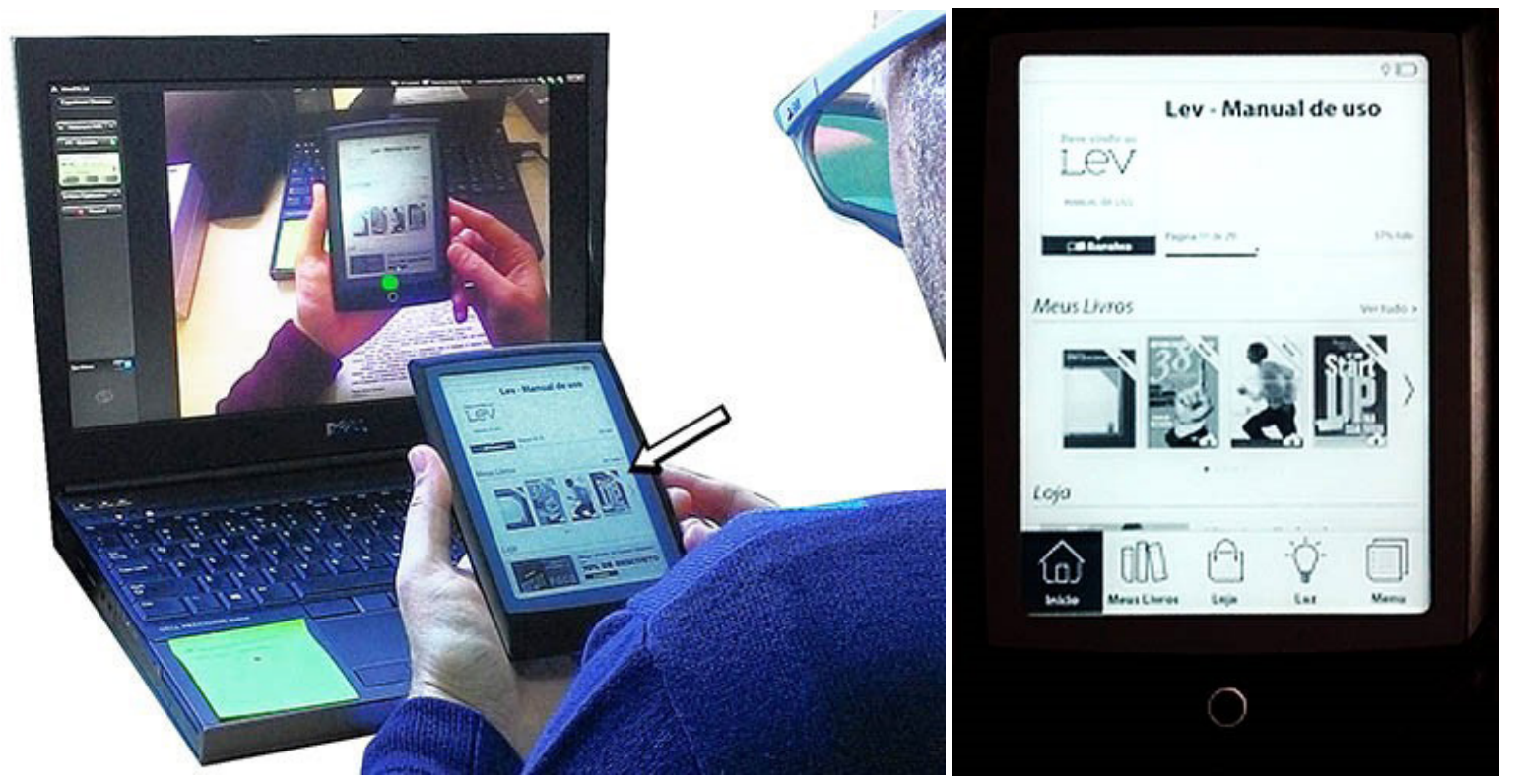

Figuras 2 e 3 - Dispositivo durante a Tarefa 1 e vista frontal do menu, dispositivo Lev. Fonte - Imagens capturadas durante 0 experimento pelos autores.

Ainda com relação a esta tarefa, a maioria dos usuários que buscavam o livro pelo preview, focava primeiramente no livro que estava em destaque, na parte superior, para depois verificar se o livro procurado estava na parte abaixo, no menu "Meus livros" (Figura 4).

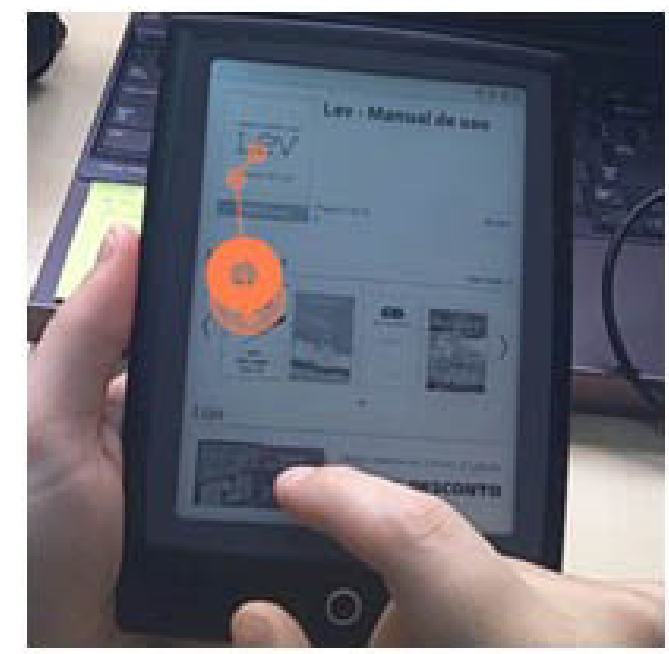

Figura 4: Caminho do olhar (scanpath) do P1 para a seleção do livro a ser aberto. Fonte: Software BeeGaze.

\subsubsection{Tarefa 2 - Zoom}

A maioria dos participantes conseguiu realizar corretamente esta tarefa por se tratar de algo mais intuitivo, utilizando o movimento de pinça (Figura 5), abrindo para aumentar a fonte e fechando para diminuí-la. Quatro dos cinco participantes que 
realizaram a tarefa com acerto parcial, primeiramente fizeram o movimento correto de pinça, entretanto, pela demora de resposta do aparelho, não perceberam que haviam mudado o tamanho da fonte e realizaram a tarefa através do menu. Apenas um participante, o único que já possuía conhecimento prévio com o aparelho similar, Kindle da Amazon, realizou a tarefa indo direto ao menu.

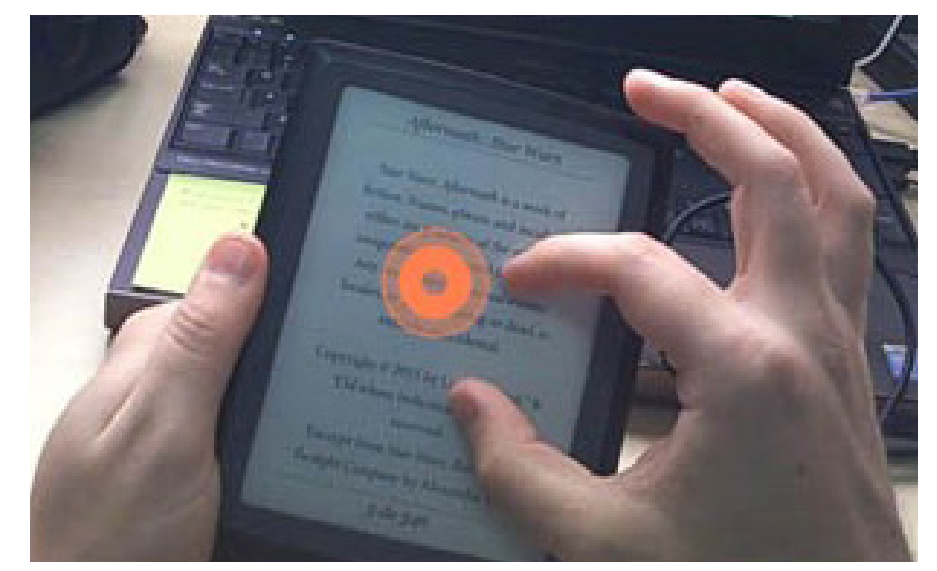

Figura 5: Exemplo de movimento de pinça, realizado pela maioria dos participantes. Fonte: Software BeeGaze.

\subsubsection{Tarefa 3 - Manual página onze}

A maioria dos participantes teve dificuldade em realizar esta tarefa da forma considerada ideal, com menos acionamentos. Nove dos onze participantes realizaram-na com acerto parcial, possivelmente pelo fato de a tarefa ter muitos passos. A maneira como eles chegavam até a página 11 não foi considerada como um problema de interface, apenas uma preferência individual. Outra questão a se levantar é que, dos seis participantes que realizaram a tarefa 1 abrindo o livro pelo preview da página inicial, três tentaram abrir o manual da mesma forma. Por não encontrá-lo no preview, houve dúvida por parte dos participantes. Isso poderia indicar que a presença dos previews na página inicial dificulta a tarefa de encontrar o livro desejado pelo usuário menos experiente. A maioria dos participantes conseguiu realizar esta tarefa indo pelo menu "Meus livros", depois selecionando "Todos os livros", e posteriormente procurando pelo manual na lista de livros do dispositivo até encontrá-lo (Figuras 6 e 7).

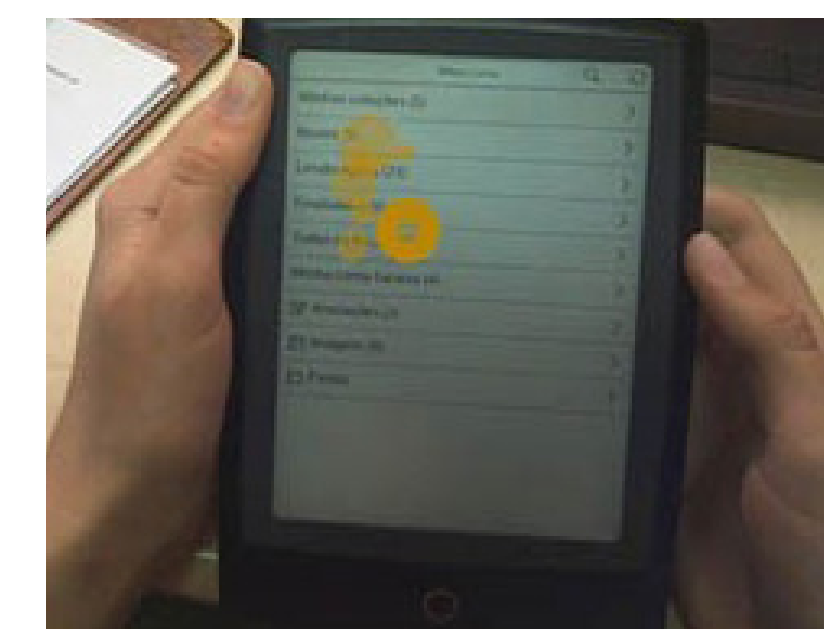

Figura 6: Caminho percorrido para chegar ao item de menu "Todos os livros". Fonte: Software BeeGaze 


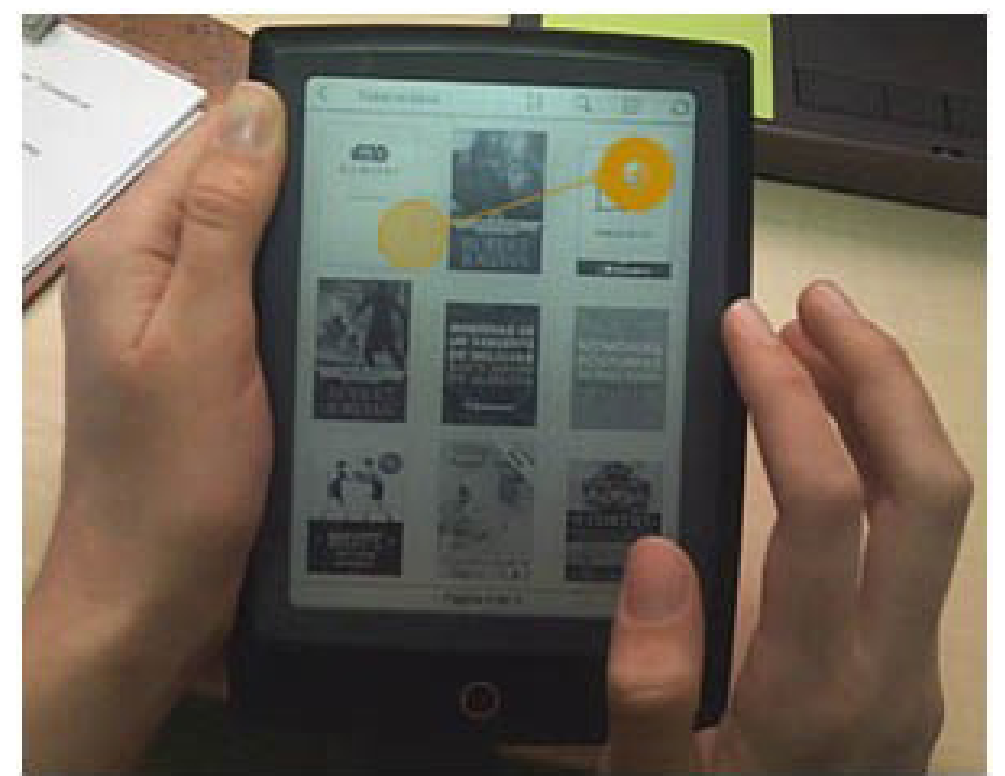

Figura 7: Participante 6 buscando o manual, passando pelos livros disponíveis no dispositivo. Fonte Software BeeGaze

\subsubsection{Tarefa 4 - Palavra no dicionário}

Mesmo sendo realizada após a leitura das orientações do manual, oito dos onze participantes realizaram esta tarefa com acerto parcial. Isso se deveu à maneira como a interface apresentava o resultado após a seleção da palavra desejada para mostrar o significado. Todos os participantes realizaram a seleção propriamente dita da forma correta, conforme orientação do manual. Porém, após a seleção da palavra, na página seguinte eram apresentadas as opções existentes de dicionários para a busca e, mais uma vez, a palavra selecionada. Esta deveria ser selecionada novamente para mostrar o seu significado. Isto gerava incerteza nos usuários, que abriam as opções dos dicionários ao invés de acionar novamente a palavra (Figura 8). 0 problema poderia ser mitigado se o programa abrisse diretamente o significado da palavra após a seleção.

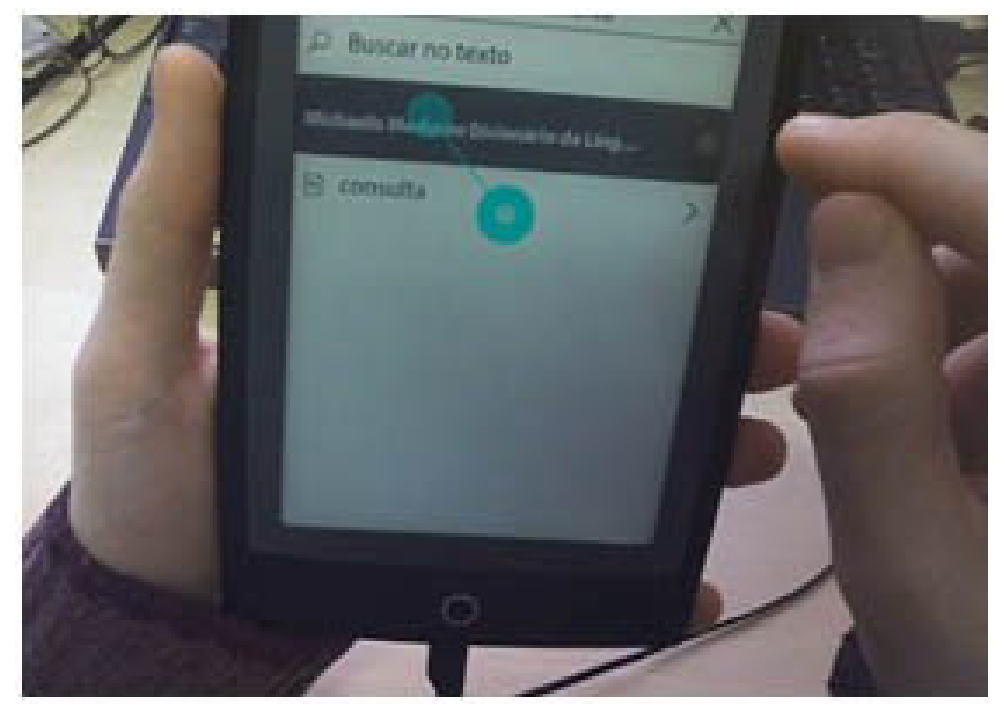

Figura 8: Participante selecionando o dicionário a ser consultado invés de acionar a palavra para a qual ele buscava o significado (palavra "consulta" no caso deste participante). Fonte: Software BeeGaze

Márcia Cattoi Schmidt, Diogo Gonçalves Martins,

Ricardo Schwinn Rodrigues, Aline Girardi Gobbi,

Giselle Schmidt Alves Diaz Merino e 


\subsubsection{Tarefa 5 - Adicionar nota}

Esta se mostrou a mais problemática das tarefas. Os participantes tiveram dificuldade na escolha da maneira de selecionar o trecho desejado. Todos tentaram selecionar o texto, pelo menos na primeira vez, da forma que seria a mais usual, acionando e segurando a primeira palavra, e deslizando o dedo sobre o texto até a última palavra (Figura 9). Os seis participantes que obtiveram falha na tarefa ficaram tentando desta forma até desistirem ou serem interrompidos pelo moderador. $O$ correto seria selecionar concomitantemente a primeira e a última palavra usando dois dedos (Figura 10).
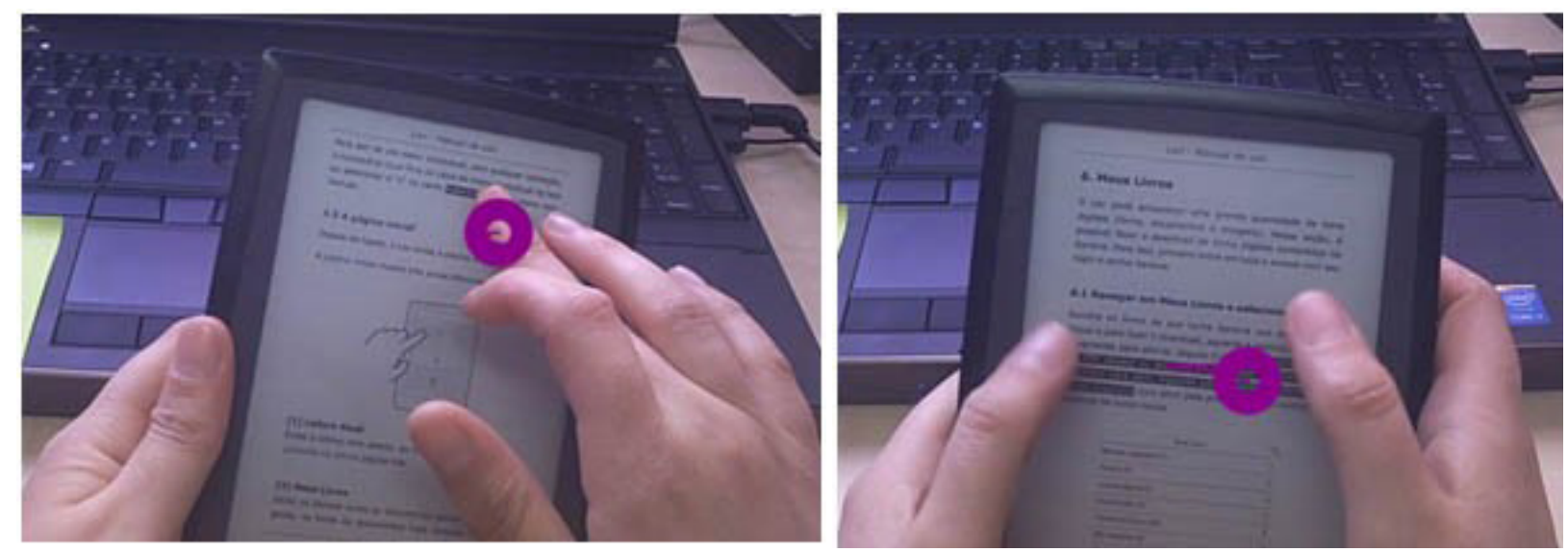

Figuras 9 e 10: Participante tentando selecionar o texto da forma mais usual e a forma correta de seleção de texto para este dispositivo usando os dois polegares. Fonte: Software BeeGaze.

\subsubsection{Tarefa 6 - Comprar um livro}

Esta se mostrou a mais fácil e intuitiva das tarefas. O único participante que a realizou com um acerto parcial retornou à página inicial e buscou a loja pelo ícone "início" contido nela, ao invés de selecionar diretamente o ícone "loja" pelo menu principal.

\subsection{Dados quantitativos}

O sistema dos óculos de rastreamento ocular (eye tracking) fornece uma série de informações relativas ao comportamento do olhar. Dentre essas informações, as mais relevantes são as fixações (onde o olhar está focado), sacadas (saltos que o olho realiza quando está percorrendo uma imagem) e as piscadas. Essas medidas podem indicar fatores como fadiga (que pode diminuir o número de piscadas), atenção (geralmente, quanto menor o número de piscadas e maior o número de fixações em uma tarefa, mais concentrado o usuário está) e vigilância (também medido pelo número de piscadas), entre outros estados mentais discutidos pela literatura (MCINTIRE, 2014). Também refletem alguns comportamentos característicos, como inquietação ou até mesmo escolhas menos diretas na realização da tarefa, prolongando o tempo do participante. Na Figura 12, percebe-se um possível problema técnico de coleta na 
amostra do participante P3, possivelmente por erro de calibração não identificado no momento da coleta. Enquanto há uma maior fixação do olhar dos outro $\neg$ s participantes, neste participante a fixação não se confirma durante a coleta pela ferramenta. (Figura 11 e 12) Este problema alterou os dados numéricos do participante P3 para mais, apenas nesta coleta quantitativa. O participante não apresentava nervosismo ou comportamento alterado, e não foi descartado porque a análise dos dados apresentou um grau de heterogeneidade considerável mesmo sem seus dados.
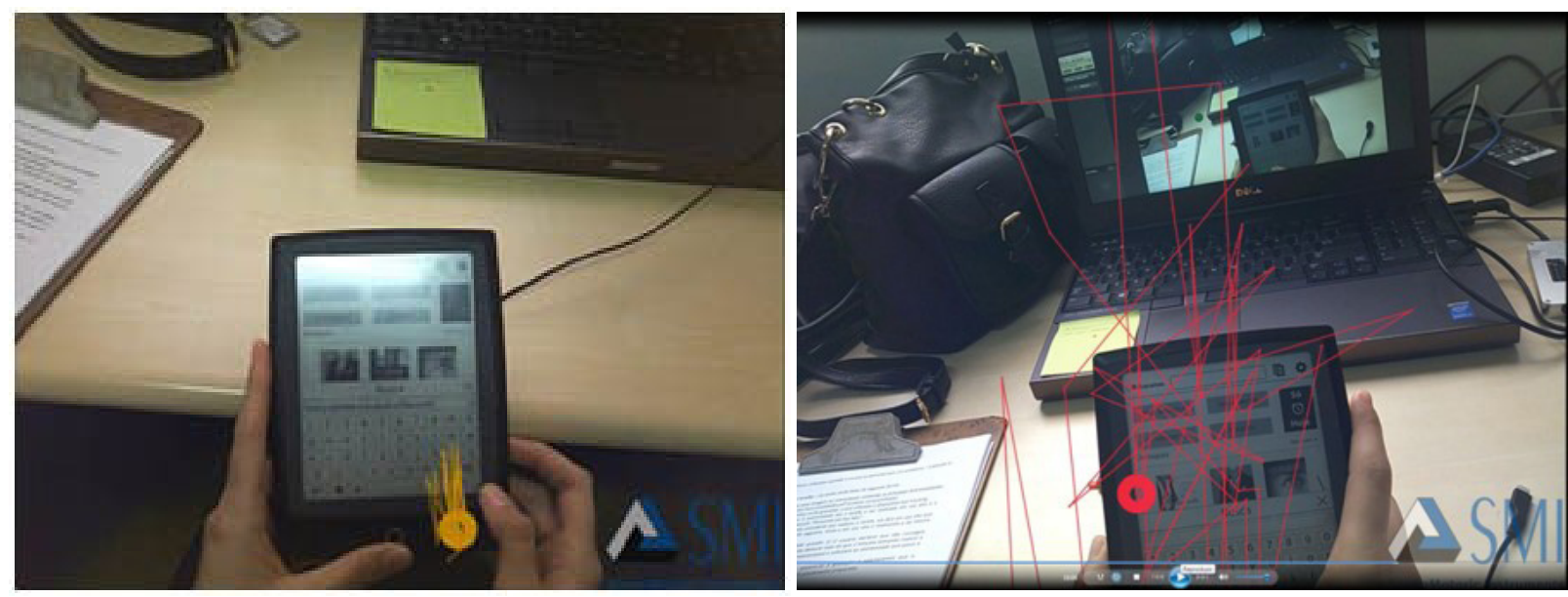

Figuras 11 e 12 - Coleta de Imagem com Eye Tracking, Participante P6 e P3 respectivamente. Fonte - Software BeeGaze.

\subsection{Questão tempo da tarefa}

O Tempo da Tarefa (em segundos) mostrou dados bastante heterogêneos, conforme a Tabela 1. É importante ressaltar que atrasos eventuais devidos à conexão com a internet na Tarefa 6 foram descontados do tempo final. Outra questão a ser analisada no quesito tempo é que alguns participantes tiveram seu tempo diminuído porque o dispositivo disponibiliza alguns livros em preview na sua página inicial, sem que se possa controlar quais, conforme explicado no item 5.1.1. Para um estudo mais preciso seria aconselhável trocar imediatamente o livro requisitado por algum livro que não apareça no preview da página inicial.

Conforme a Tabela 1, os números em vermelho marcam onde os participantes necessitaram ajuda do moderador para completá-la, apontando as Tarefas 3 e 5 como complexas ou problemáticas. Quanto maior o desvio padrão DP, maior a dispersão dos dados, sendo que a tarefa 2 é a mais homogênea e a tarefa 3 é a mais heterogênea na questão tempo. Percebem-se os picos de tempo nas Tarefas 3 e 5 , embora alguns participantes as tenham resolvido com tempos similares às outras tarefas, como o Participante 9, por exemplo. 


\begin{tabular}{ccccccc}
\hline & $\begin{array}{c}\text { Tarefa 1 } \\
\text { (encontrar } \\
\text { livro) }\end{array}$ & $\begin{array}{c}\text { Tarefa 2 } \\
\text { (zoom) }\end{array}$ & $\begin{array}{c}\text { Tarefa 3 } \\
\text { (manual } \\
\text { página 11) }\end{array}$ & $\begin{array}{c}\text { Tarefa 4 } \\
\text { (palavra no } \\
\text { dicionário) }\end{array}$ & $\begin{array}{c}\text { Tarefa 5 } \\
\text { (adicionar } \\
\text { nota) }\end{array}$ & $\begin{array}{c}\text { Tarefa 6 } \\
\text { (comprar } \\
\text { livro) }\end{array}$ \\
\hline P1 & 97 & 43 & 175 & 95 & $\mathbf{1 5 8}$ & 68 \\
\hline P2 & 81 & 8 & 43 & 13 & $\mathbf{1 0 6}$ & 61 \\
\hline P3 & 9 & 4 & $\mathbf{1 8 9}$ & 15 & $\mathbf{1 5 8}$ & 137 \\
\hline P4 & 42 & 29 & 146 & 27 & 52 & 70 \\
\hline P5 & 8 & 29 & 155 & 28 & 79 & 94 \\
\hline P6 & 38 & 9 & 95 & 38 & $\mathbf{4 7}$ & 81 \\
\hline P7 & 1 & 46 & 141 & 36 & $\mathbf{1 3 8}$ & 58 \\
\hline P8 & 10 & 35 & 153 & 17 & $\mathbf{5 8}$ & 133 \\
\hline P9 & 22 & 43 & 60 & 24 & 53 & 19 \\
\hline P10 & 12 & 19 & 23 & 4 & 143 & 86 \\
\hline P11 & 9 & 33 & 104 & 44 & 154 & 54 \\
\hline Média & 29,9 & $\mathbf{2 7 , 1}$ & $\mathbf{1 1 6 , 7}$ & $\mathbf{3 1}$ & $\mathbf{1 0 4 , 2}$ & $\mathbf{7 8 , 3}$ \\
DP & $\mathbf{3 2 , 1}$ & $\mathbf{1 5}$ & $\mathbf{5 5 , 6}$ & $\mathbf{2 4 , 3}$ & $\mathbf{4 7 , 2}$ & $\mathbf{3 4 , 3}$ \\
\hline \multicolumn{7}{c}{ Tabela 1-Tabela de tempo em segundos das Tarefas por Participante. } \\
\end{tabular}

\subsection{Questionário sus}

O System Usability Scale (SUS), desenvolvido por John Brooke em 1986 (TULLIS; ALBERT, 2013, P.137), foi utilizado para a obtenção de dados subjetivos acerca da satisfação percebida por cada participante durante o uso do Lev. O SUS é composto por 10 questões (valoradas pelo usuário de 1 a 5) que abordam diferentes aspectos da usabilidade como: facilidade de uso, consistência, complexidade no uso, entre outros. A escala gera dados quantitativos em uma pontuação que vai de 0 a 100. Utilizaram-se as pontuações apresentadas por Tullis e Albert (2013, p. 139) como parâmetro no presente estudo, sendo estas <50: Não aceitável; 50-70: Mediano; >70: Aceitável. A pontuação SUS obtida por cada participante desde estudo é apresentada na Figura 13 a seguir.

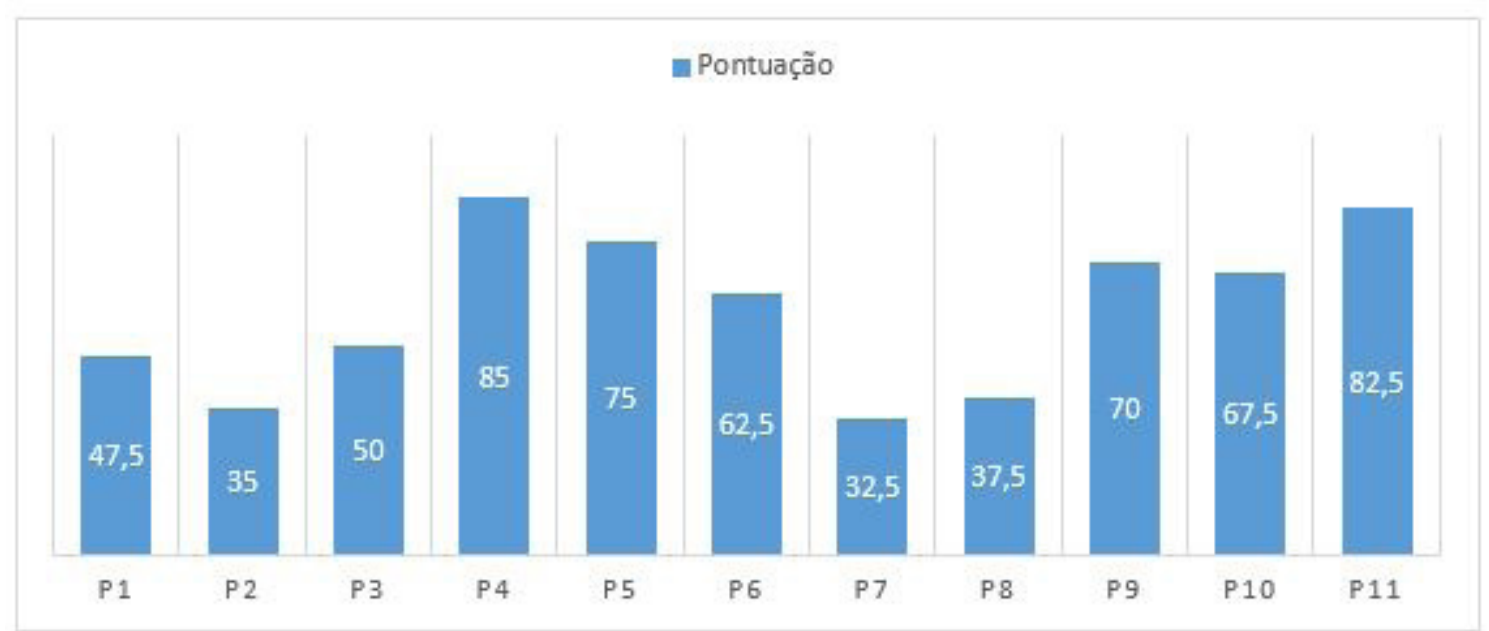

Figura 13 - Gráfico de pontuação SUS (System Usability Scale). Fonte - Elaborado pelos autores, conforme dados coletados. 
A média geral da pontuação SUS obtida nas respostas dos 11 participantes é de 58,63 com desvio padrão (DP) de 19,08, considerado um nível de satisfação mediano de acordo com a escala de Tullis e Albert (2013). Entre os participantes, quatro geraram pontuação menor do que 50 e apenas três geraram pontuação acima de 70, ou seja, estavam satisfeitos com a interface.

A pontuação SUS de 58,63 obtida pelo dispositivo Lev foi, desta forma, inferior à apresentada por e-readers em outros estudos. Gatsou et al (2015) testaram a usabilidade do e-reader Cybook Odyssey e obtiveram a pontuação SUS 70,8. Este e-reader possui tecnologia e-ink e mesmo tamanho de tela do Lev. Em seus experimentos, doze usuários novatos realizaram tarefas semelhantes ao do presente estudo, como abrir livros, fazer marcações, criar notas, aumentar e diminuir o tamanho de texto. Jardina e Chaparro (2012), por sua vez, testaram a usabilidade de e-readers com telas coloridas, com dezesseis usuários novatos obtendo média da pontuação SUS de 64,2 $(\mathrm{DP}=18,9)$ para o Kindle Fire e 66.7 (DP $=19,7$ ) para o Nook Tablet. Vale ressaltar que os experimentos de Gatsou et al (2015) e Jardina e Chaparro (2012) apresentaram tarefas semelhantes aos deste estudo e Gatsou et al (2015) testou um e-reader com especificações similares ao Lev.

A satisfação mediana obtida no presente estudo pode ser consequência do tempo de processamento elevado do e-reader Lev, e da baixa taxa de resposta da tecnologia e-ink, que podem frustrar o usuário. Os participantes realizaram múltiplos toques durante o experimento, ao acreditar que o Lev não havia reconhecido sua ação, quando na verdade, a ação ainda estava sendo processada pelo dispositivo. 0 fato da pontuação SUS ter sido inferior ao estudo de Gatsou et al (2015), que testou um dispositivo com hardware semelhante, pode indicar que o ponto crítico da usabilidade esteja relacionada com o gestual necessário para realizar as ações. A prova disso está no fato dos usuários considerarem pouco intuitivos os gestos de selecionar trechos de texto, pois a sistemática da ação é diferente da adotada por outros sistemas operacionais com telas de toque a que estavam habituados.

Após a realização dos testes, solicitou-se aos participantes que respondessem, de forma discursiva, as seguintes perguntas: Qual tarefa você achou mais fácil de executar e por quê? Qual tarefa você achou mais difícil e por quê? Abaixo segue a Tabela 2 com as frequências em que cada tarefa foi respondida pelos participantes, para cada pergunta.

\begin{tabular}{lcc}
\hline & Frequência de respostas para tarefas consideradas: \\
\cline { 2 - 3 } & FÄCEIS & DIFiCEIS \\
Tarefa 1 (encontrar livro) & 4 & 0 \\
Tarefa 2 (zoom) & 3 & 2 \\
Tarefa 3 (manual página 11) & 2 & 1 \\
Tarefa 4 (paláfico \\
dicionário) & 0 & 8 \\
Tarefa 5 (adicionar nota) & & 0 \\
Tarefa 6 (comprar livro) & 2 & \\
\hline
\end{tabular}

Tabela 2 - Percepção de facilidade e dificuldade na tarefa. Fonte: Elaborada pelos autores, conforme dados coletados. 
Houve frequências similares nas respostas das tarefas consideradas mais fáceis pelos participantes. A tarefa 1 e 2 foram as mais frequentes, e portanto mais fáceis, com 4 e 3 respostas respectivamente. A segunda parte da pergunta, que identifica o porquê da tarefa ser considerada a mais fácil, trouxe justificativas bastante similares para cada tarefa. A Tarefa 1 foi considerada a mais fácil provavelmente porque para alguns usuários a imagem da capa do livro estava visível na página inicial do Lev. Um participante achou fácil encontrar o livro, pois a interface apresenta "setas de rolagem" para navegação na biblioteca, o que foi considerado similar à interface de outros sistemas operacionais. A Tarefa 2 foi considerada a segunda tarefa mais fácil devido à similaridade em realizar o gesto de zoom do Lev com outros dispositivos móveis com tela de toque, sendo considerada intuitiva pelos participantes.

A tarefa que apareceu em maior frequência como sendo a mais difícil foi a Tarefa 5, com oito respostas. Deste total, seis participantes consideraram a tarefa difícil, pois o gesto utilizado para selecionar as palavras é diferente do adotado em outros sistemas operacionais, e dois participantes justificaram a escolha pela pouca intuitividade na execução da tarefa.

Finalmente, foi solicitado aos participantes manifestarem as suas impressões gerais relativas à usabilidade do sistema, de forma discursiva. Oito participantes consideraram o sistema lento. Destes, dois apontaram que isto causou frustração durante o uso e um apontou que não sabia se havia realizado a tarefa, devido ao atraso na resposta do dispositivo. Quatro participantes compararam a Tarefa 5 (considerada a mais difícil) com smarthphones e tablets e argumentaram que a tarefa é pouco intuitiva, pois o gestual necessário para executá-la diverge de outros sistemas operacionais, necessitando de tempo para aprender este novo modo de execução. Apesar das discrepâncias, os participantes destacaram a simplicidade do sistema que, mesmo sendo pouco intuitivo, é de rápido aprendizado.

Em suma, identificou-se na análise das respostas subjetivas dos participantes, que a comparação do Lev com outros dispositivos móveis como smarthphones e tablets, foi o fator decisivo para a percepção das tarefas executadas. Portanto, uma tarefa foi considerada fácil quando as ações necessárias para desempenhá-la eram similares às de outros sistemas, caso contrário, a tarefa foi considerada difícil.

\section{Conclusão}

Ao explorar a experiência de onze usuários potenciais, no uso intuitivo do produto e-reader LEV (Saraiva), procurou-se identificar problemas de interface no produto. $O$ experimento realizado permitiu o levantamento de aspectos positivos e negativos da usabilidade do e-reader Lev, bem como o nível de satisfação dos usuários na utilização do dispositivo.

O dispositivo de Eye-tracking tem colaborado em testes de usabilidade atualmente, associado a outras técnicas de análise de produtos. A aplicação desta ferramenta colaborou para a coleta massiva de dados, demandando um trabalho de decodificação intenso, que ainda pode ser retomado e ampliado. Para trabalhos futuros, podem ser feitas comparações entre tecnologias de leitores digitais, compara-

ções entre faixas etárias, ou novas funcionalidades podem ser testadas, como o uso 
da lanterna do dispositivo em ambiente escurecido, a formação de coleções, entre outras.

Considerados os dados provenientes do experimento, e questionários pós-teste, pode-se dizer que o ponto crítico na usabilidade no aparelho está na pouca intuitividade da interface. Isto pode ser observado na dificuldade dos participantes na realização das tarefas que traziam elementos particulares deste sistema, como a busca de palavras em dicionários e seleção de trechos de textos que exigem comandos específicos não comumente utilizados em interfaces móveis. A usabilidade também é prejudicada pelo tempo de resposta do dispositivo em comparação à rapidez dos outros dispositivos - smartphones e tablets, causando irritação e frustração nos participantes pela repetição da ação até funcionar.

\section{AGRADECIMENTOS}

Os autores gostariam de agradecer a dedicação e apoio de Aline G. Gobbi, Giselle Merino, Eugênio D. Merino e todos os participantes da pesquisa. Esta pesquisa foi realizada por uma parceria entre a Universidade do Estado de Santa Catarina/ UDESC e a Universidade Federal de Santa Catarina/UFSC.

\section{Referências}

ALBERT, William; TULLIS, Thomas. Measuring the user experience: collecting, analyzing, and presenting usability metrics. Newnes, 2013.

CUNHA, Joana. Mercado de livros digitais não decola no Brasil e estagna nos EUA e Europa. 2016. Disponível em: < http://www1.folha.uol.com.br/mercado/2016/04/1759174-mercado-de-livros-digitais-nao-decola-no-brasil-e-estagnanos-eua-e-europa.shtml >. Acessado em: 2 de julho, de 2016.

GATSOU, Chrysoula; POLITIS, Anastasious; ZEVGOLIS, Dimitrios. Exploring Users' Experience With E-Reading Devices. Proceedings of the Federated Conference on Computer Science and Information Systems pp. 833-839; 2015, IEEE.

JARDINA, Jo R.; CHAPARRO, Barbara S. Usability of e-readers for book navigation tasks. In: Proceedings of the Human Factors and Ergonomics Society Annual Meeting. SAGE Publications, 2012. p. 1897-1901.

LARSON, Lotta C. Electronic Reading Workshop: Beyond Books With New Literacies and Instructional Technologies, 2008.

MCINTIRE, Lindsey $\mathrm{K}$. et al. Detection of vigilance performance using eye blinks. Applied ergonomics, v. 45, n. 2, p. 354-362, 2014. 
NIELSEN, Jacob. iPad and Kindle Reading Speeds. 2010. Disponível em: < https:// www.nngroup.com/articles/ipad-and-kindle-reading-speeds/\# >. Acessado em: 2 de julho, de 2016.

O GLOBO. Vendas de livros impressos sobem, enquanto digitais perdem popularidade, diz 'FT'. 2015. Disponível em: < http://oglobo.globo.com/economia/negocios/ vendas-de-livros-impressos-sobem-enquanto-digitais-perdem-popularidade-diz -ft-15020531 >. Acessado em: 2 de julho, de 2016.

RUBIN, Jeffrey; CHISNELL, Dana - Handbook Of Usability Testing How To Plan, Design, And Conduct Effective Tests - 2ed., Wiley Publishing, Inc., Indianapolis, Indiana, 2008.

PRECE, Jenny; ROGERS, Yvonne; SHARP, Helen. INTERACTION' DESIGN beyond human-computer interaction. John Wiley \& Sons, Inc, NY, 2002.

SIEGENTHALER, Eva, SCHMID, Laura, WYSS, Michael, WURTZ, Pascal. LCD vs. E-ink: An Analysis of the Reading Behavior. Journal of Eye Movement Research, 2012.

SIEGENTHALER, Eva, WURTZ, Pascal, BACHUD, Yves, BERGAMIN, Per. Reading on $L C D$ versus e-Ink displays: Effects on fatigue and visual strain. Ophthalmic and Physiological Optics, 2010.

SIEGENTHALER, Eva, WURTZ, Pascal, BERGAMIN, Per, GRONER, Rudolf. Comparing reading processes on e-ink displays and print. Displays, 2011.

TULLIS, Tom; ALBERT, Bill. Measuring the User Experience, Collecting, Analyzing, and Presenting Usability Metrics. Morgan Kaufmann, 2008.

ZAMBARBIERI, Daniela, CARNIGLIA, Elena. Eye movement analysis of reading from computer displays, eReaders and printed books. Ophthalmic \& Physiological Optics, 2012.

INSTITUTO PRÓ-LIVRO IPL. Retratos da Leitura no Brasil 4aㅡ Ed, São Paulo, 2016. Disponível em: < http://prolivro.org.br/home/images/2016/Pesquisa_Retratos_da_Leitura_no_Brasil_-_2015.pdf >. Acessado em: 2 de julho, de 2016. 


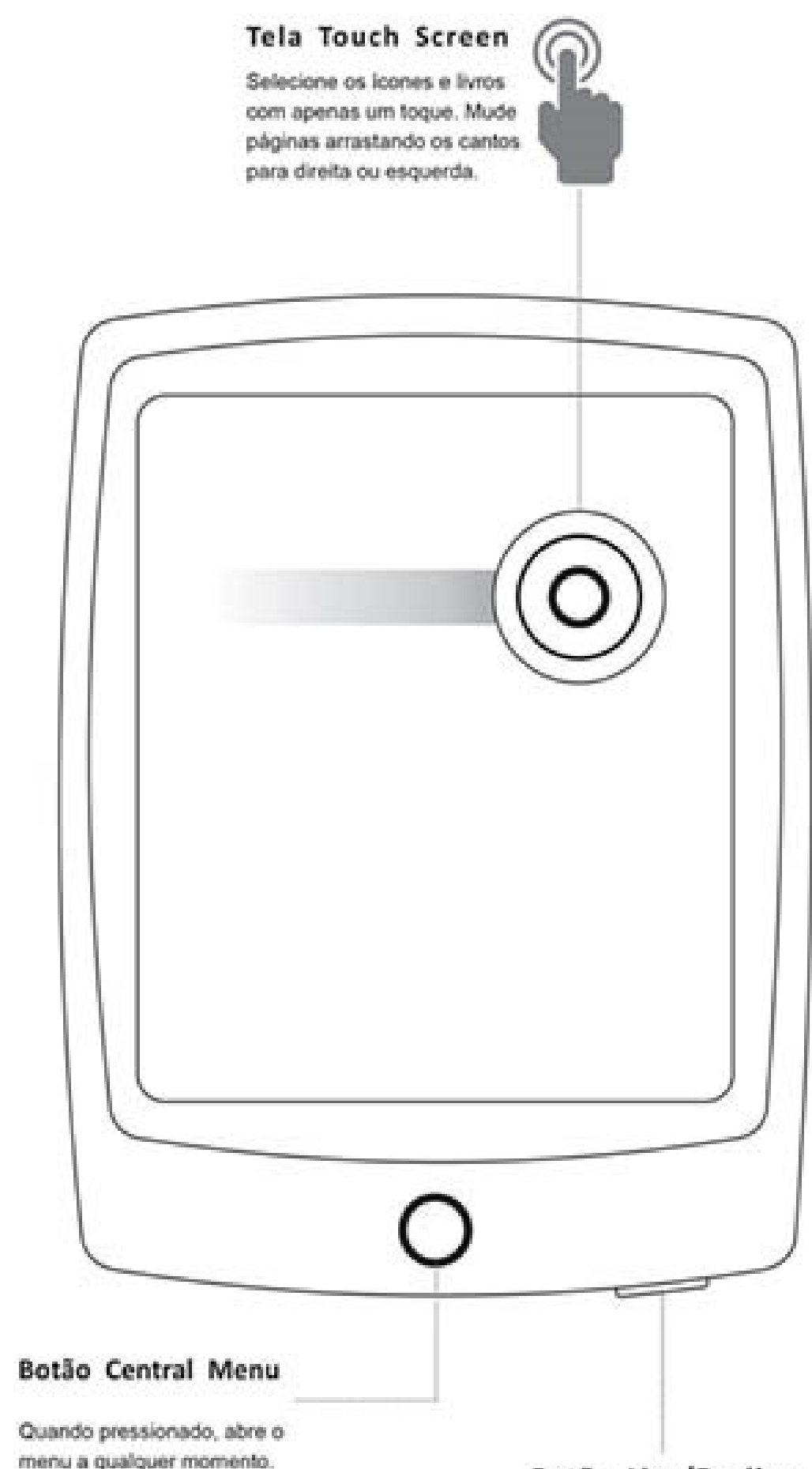

menu a qualquer momento.

Botão Liga/Desliga

Quando pressionado, liga

ou desliga o apareiho.

Márcia Cattoi Schmidt, Diogo Gonçalves Martins,

Ricardo Schwinn Rodrigues, Aline Girardi Gobbi,

Giselle Schmidt Alves Diaz Merino e 